\title{
ON THE GROUPS OF COBORDISM $\Omega^{k}$
}

\author{
MASAHISA ADACHI
}

\section{Introduction}

In the papers [11] and [18] Rohlin and Thom have introduced an equivalence relation into the set of compact orientable (not necessarily connected) differentiable manifolds, which, roughly speaking, is described in the following manner: two differentiable manifolds are equivalent (cobordantes), when they together form the boundary of a bounded differentiable manifold. The equivalence classes can be added and multiplied in a natural way and form a graded algebra $\Omega$ relative to the addition, the multiplication and the dimension of manifolds. The precise structures of the groups of cobordism $\Omega^{k}$ of dimension $k$ are not known thoroughly. Thom [18] has determined the free part of $\Omega$ and also calculated explicitly $\Omega^{k}$ for $0 \leqq k \leqq 7$.

The purpose of the present paper is to determine explicitly the groups $\Omega^{k}$ for $8 \leqq k \leqq 12$. Our method is analogous to that of Thom [18] and we shall calculate $\Omega^{k}$ using Serre's $C$-theory.

In $\S 1$ we explain shortly some general results on the Eilenberg-MacLane complexes, Serre's $C$-theory and the Grassmann manifold, which will be used later. In $\S 2$ the homotopy groups of the Thom complex $M(S O(n))$ associated with the rotation group are calculated. In $\S 3$ we determine the groups of cobordism $\Omega^{k}$ for $8 \leqq k \leqq 12$, and discuss some problems related to $\Omega^{k}$.

Some of the results contained in this paper have been announced in the note [1].

The author is deeply grateful to Professors R. Shizuma and N. Shimada for their kind encouragements and valuable criticisms.

\section{$\S 1$. Preliminaries}

Before we approach the determination of the homotopy groups of the Thom complex $M(S O(n))$ associated with the rotation group, it is necessary to recall

Received December 14, 1957.

Revised March 24, 1958. 
some general results on the reduced powers of Steenrod, the Eilenberg-MacLane complexes, Serre's $C$-theory and the Grassmann manifolds.

We shall denote by $Z$ and $Z_{p}$ the ring of integers and of integers modulo $p$ respectively.

\section{A. Reduced powers of Steenrod}

Let $p$ be a prime number. The Bockstein homomorphism $\alpha_{p}: H^{i}\left(X, Z_{p}\right)$ $\rightarrow H^{i+1}(X, Z)$ is identical with $(-1)^{i+1} \delta$, where $\delta$ is the coboundary homomorphism of the cohomology exact sequence of a space $X$ relative to the exact sequence of coefficient groups

$$
0 \rightarrow Z \rightarrow Z \rightarrow Z_{p} \rightarrow 0 .
$$

The Bockstein homomorphism $\beta_{p}: H^{i}\left(X, Z_{p}\right) \rightarrow H^{i+1}\left(X, Z_{p}\right)$ is defined by the composition of $\alpha_{p}$ and the natural homomorphism

$$
\rho_{p}: H^{i+1}(X, Z) \rightarrow H^{i+1}\left(X, Z_{p}\right) .
$$

Let $a$ be an integer $\geq 0$ congruent to 0 or $1 \bmod 2 p-2$. We define the homomorphism

$$
S t_{p}^{a}: H^{i}\left(X, Z_{p}\right) \rightarrow H^{i+a}\left(X, Z_{p}\right)
$$

in the following manner: if $p=2$, we put $S t_{p}^{a}=S q^{a}$; if $p>2$ and $a=2 k(p-1)$, $k$ an integer, we put $S t_{p}^{a}=P_{p}^{k}$; if $p>2$ and $a=2 k(p-1)+1, S t_{p}^{a}=\beta_{p} \circ P_{p}^{k}$. For a sequence $I=\left(a_{1}, a_{2}, \ldots, a_{r}\right)$ of integers $a_{i} \geq 0$, congruent to 0 or $1 \mathrm{mod}$ $2 p-2$, we denote the composed operation by

$$
S t_{p}^{l}=S t_{p}^{a_{1}} \circ S t_{p}^{a_{2}} \circ \ldots \circ S t_{p}^{a_{r}} .
$$

The following formulas are often used in $\S 2$.

$$
\begin{gathered}
P_{p}^{k}(u \cdot v)=\sum_{i=0}^{k} P_{p}^{i}(u) P_{p}^{k-i}(v), \\
P_{p}^{k} \circ \beta_{p} \circ P_{p}^{h}=\left(\begin{array}{c}
k+h-1 \\
h
\end{array}\right) P_{p}^{k+h} \circ \beta_{p}+\left(\begin{array}{c}
k+h-1 \\
k
\end{array}\right) \beta_{p} \circ P_{p}^{k+h}, \\
\text { for } 0 \leqq k \leqq p-1,
\end{gathered}
$$

where $\quad\left(\begin{array}{l}a \\ b\end{array}\right)=\left\{\begin{array}{l}\text { binomial coefficient reduced } \bmod p \text {, if } a, b \leqq 0, \\ 0, \text { if } a<0 \text { or } b<0,\end{array}\right.$

(cf. Cartan [5]). 


\section{B. Eilenberg-MacLane complexes}

Let $n$ be an integer $\triangleq 1$, and $\Pi$ be an abelian group. An arcwise connected space $X$ is called the Eilenberg-MacLane complex $K(\Pi, n)$ if all its homotopy groups of dimension $>0$ are zero except for $\pi_{n}(X)=\Pi$. All these spaces have the same homotopy type, and among them there exists a simplicial complex. Moreover,

1) If $\Pi$ is an abelian group of finite type, there exists a simplicial complex $K(\Pi, n)$ whose $q$-skelton is a finite complex (cf. Thom [18], p. 36).

We denote the cohomology ring of $K(\Pi, n)$ with coefficients in $G$ by the notation $H^{*}(\Pi, n ; G)$; the group $H^{n}(G, n ; G)$ possesses a fundamental class which we will denote by $\iota_{n}$.

2) For any cohomology class $u \in H^{n}(X, G)$ of a topological space $X$, there exists a mapping $f: X \rightarrow K(G, n)$ such that $u=f^{*}\left(\iota_{n}\right)$.

The cohomology of complexes $K(Z, n)$ and $K\left(Z_{p}, n\right)$ has been determined by Cartan [4] and Serre [14]. Here let us recall some of their results.

3) The cohomology ring $H^{*}\left(Z_{2}, n ; Z_{2}\right)$ is generated by the Steenrod squarings of the fundamental class $\mathrm{n}_{n} \in H^{n}\left(Z_{2}, n ; Z_{2}\right)$ and their cup products; for $h<n$ (stable part of $H^{*}\left(Z_{2}, n ; Z_{2}\right)$ ), a base of the group $H^{n+h}\left(Z_{2}, n ; Z_{2}\right.$ ) is given by the sequences of iterated squarings of $\iota_{n}: S q^{I}\left(\ell_{n}\right)$, where $I=\left(i_{1}, i_{2}\right.$, $\left.\ldots, i_{r}\right)$ with $\sum_{m} i_{m}=h$ and $i_{s} \geq 2 i_{s+1}$, for $1 \leqq s \leqq r-1$.

We have an analogous result for $H^{*}\left(Z, n ; Z_{b}\right)$.

4) For $h<n$, a base of $H^{n+h}\left(Z, n ; Z_{p}\right)$ is given by the sequences of iterated reduced powers (squarings if $p=2) S t_{p}^{\prime}\left(\ell_{n}\right)$, where $I=\left(a_{1}, a_{2}, \ldots, a_{r}\right)$ satisfying the following conditions: ${ }^{11}$

$$
\begin{aligned}
& a_{i} \equiv 0 \text { or } 1, \bmod 2 p-2, \text { for } 0 \leqq i \leqq r, \\
& a_{i} \geqq p a_{i+1}, \text { for } 1 \leqq i \leqq r-1, \\
& a_{r} \geqq 2 p-2, \\
& \sum_{i} a_{i}=h .
\end{aligned}
$$

\section{Serre's $C$-theory}

Let $C_{p}$ be the class of finite abelian groups whose $p$-primary components are zero, where $p$ is a prime number. We shall often use the following theorem in $\S 2$.

1) We denote by $l_{n}$ also the fundamental class of $K(Z, n)$ reduced mod $p$. 
THEOREM. Let two spaces $A$ and $B$ be connected and simply arcwise connected, $f: A \rightarrow B$ be a mapping which maps $\pi_{2}(A)$ onto $\pi_{2}(B)$ and $n$ be $a$ positive integer. Suppose the homology groups $H_{\imath}(A, Z)$ and $H_{i}(B, Z)$ are finite type in all dimensions. Then the following two properties are equivalent:

a) $f_{*}: \pi_{i}(A) \rightarrow \pi_{i}(B)$ is $C_{p}$-isomorphism for $i<n$, and $C_{p^{-}}$onto for $i \leqq n$.

b) $f^{*}: H^{i}\left(B, Z_{p}\right) \rightarrow H^{i}\left(A, Z_{p}\right)$ is an isomorphism for $i \leqq n$, and onto for $i<n$.

(Serre [13], Chapitre III, Théorème I and Proposition 2).

Let $G$ and $H$ be abelian groups. We denote " $G$ is $C$-isomorphic to $H$ " by $G \cong H \bmod C$.

\section{Some formulas in Grassmann manifold}

We donote by $\hat{G}_{n}$ the Grassmann manifold of oriented $n$-spaces in a Euclidean $s$-space $R^{s}$ where $s$ is sufficiently large. It is well known that $\hat{G}_{n}$ is the classifying space $B_{S O(n)}$ associated with the rotation group $S O(n)$.

1) We know that the cohomology ring $H^{*}\left(\hat{G}_{n}, Z_{2}\right)$ is a polynomial algebra generated by $W^{2}, W^{3}, \ldots, W^{n}$, where $W^{i}$ is the $i$-dimensional Stiefel-Whitney class (Borel [2]).

2) Let $n$ be even, $n=2 m$, and $p$ be an odd prime. The cohomology ring $H^{*}\left(\hat{G}_{n}, Z_{n}\right)$ is a polynomial algebra generated by the Pontrjagin classes mod $p$ and the Euler-Poincaré class mod $p$ :

$$
P^{4}, P^{8}, \ldots, P^{4 m-4}, X^{n}
$$

(Borel-Serre [3]).

3) The following formula, which gives the Steenrod squarings of $W^{i}$, was introduced by $\mathrm{Wu}[20]$ :

$$
S q^{i} W^{j}=\sum_{t=0}^{i}\left(\begin{array}{c}
j-i+t-1 \\
t
\end{array}\right) W^{i-t} W^{j+t}, \quad(i \leqq j),
$$

with the following conventions

$$
\left(\begin{array}{l}
a \\
b
\end{array}\right)=\left\{\begin{array}{l}
\text { binomial coefficient reduced } \bmod 2, \text { if } a \geqslant b>0 \\
1, \text { if } b=0, \\
0, \text { if } b \neq 0, a<b
\end{array}\right.
$$

and $W^{1}=0$.

4) It is often useful to consider the Pontrjagin classes mod $p$ and the 
Euler-Poincaré class mod $p$ of the universal bundle $E_{\mathrm{S} O(n)} \rightarrow B_{S O(n)}$ as the symmetric functions ${ }^{2)}$ of $m$ variables $x_{1}, x_{2}, \ldots, x_{m}$ of degree 2 :

$$
\begin{array}{rlrl}
P^{4 i} & =\sum x_{1}^{2} x_{2}^{2} \ldots x_{i}^{2}, & & 1 \leqq i \leqq m-1, \\
X^{n}=x_{1} x_{2} \ldots x_{m}, & n=2 m,
\end{array}
$$

where $x_{1}, x_{2}, \ldots, x_{m}$ are generators of $H^{*}\left(B_{T}, Z_{p}\right)(T$ is the maximal torus of the rotation group $S O(n))$. The introduction of the variables $x_{i}$ leads us to the following formulas:

i) $P_{p}^{k}\left(X^{n}\right)=X^{n} \sum x_{1}^{2 h} x_{2}^{2 h} \ldots x_{k}^{2 h}, \quad$ where $h=\frac{p-1}{2}$,

ii) $P_{p}^{k}\left(P^{4 i}\right)=P_{p}^{k}\left(\sum x_{1}^{2} \ldots x_{i}^{2}\right)$

$$
=\sum_{2 r+s=k} 2^{s} \sum x_{1}^{2 p} x_{2}^{2 p} \ldots x_{r}^{2 p} \cdot x_{r+1}^{p+1} \ldots x_{r+s}^{p+1} \cdot x_{r+s+1}^{2} \ldots x_{i}^{2}
$$

(Borel-Serre [3]).

5) Here we adopt Hirzebruch's definition of Pontrjagin classes (Hirzebruch $[8]$, p. 67). Then we have

$$
\rho_{2}\left(P^{4 i}\right)=\left(W^{2 i}\right)^{2},
$$

where $\rho_{2}: H^{*}\left(\hat{G}_{n}, Z\right) \rightarrow H^{*}\left(\hat{G}_{n}, Z_{2}\right)$ is the reduction $\bmod 2$.

\section{§2. Homotopy groups of Thom complex $M(S O(n))$}

In this section we shall calculate the stable homotopy groups of the Thom complex $M(S O(n))$ associated with the rotation group $S O(n)$.

\section{A. Thom complexes}

We know that any $(n-1)$-sphere bundle over a finite complex whose structure group is the rotation group $S O(n)$ is induced from the universal sphere bundle $p: E_{S O(n)}^{\prime} \rightarrow B_{S o(n)}$. We denote by $A_{S o(n)}$ the mapping cylinder of the projection $p$; this is a manifold with boundary $E_{s o(n)}^{\prime}$; we denote by $A_{S O(n)}^{\prime}$ the complement $A_{s o(n)}-E_{S O(n)}^{\prime}$ of the boundary in $A_{s o(n)}$.

We call the complex obtained from $A_{s o(n)}$ by the identification of its boundary $E_{S o(n)}$ to a point a Thom complex associated with the rotation group $S O(n)$; we shall denote it by $M(S O(n))$. Then $M(S O(n))$ is the Alexandroff's compactification of $A_{S o(n)}^{\prime}$.

2) In the present paper, we denote a symmetric function by its initial term preceded with $\Sigma$. 
The homotopy groups and the cohomology rings of Thom complex $M(S O(n))$ have been studied by Thom [18].

1) The homotopy groups $\pi_{n+k}(M(S O(n)))$ are independent on $n$, for $n>k$ (Thom [18], Théorème II. 7).

Owing to 1), hereafter we assume $n$ to be sufficiently large and even without loss of generality.

2) $\pi_{1}(M(S O(n)))=0$.

3) The cohomology ring $H^{*}\left(M(S O(n)), Z_{2}\right)$ of $M(S O(n))$ is isomorphic to the ideal generated by the $n$-dimensional Stiefel-Whitney class $W^{n}$ in the polynomial algebra $H^{*}\left(\hat{G}_{n}, Z_{2}\right)=Z_{2}\left[W^{2}, W^{3}, \ldots, W^{n}\right]$.

4) Let $p$ be an odd prime. The cohomolohy $\operatorname{ring} H^{*}\left(M(S O(n)), Z_{p}\right)$ of $M(S O(n))$ is isomorphic to the ideal generated by the Euler-Poincaré class $\bmod p X^{n}$ in the polynomial algebra $H^{*}\left(\hat{G}_{n}, Z_{p}\right)=Z_{p}\left[P^{4}, P^{8}, P^{4 m-4}, X^{n}\right]$, where $n=2 m$.

5) The cohomology ring $H^{*}(M(S O(n)), Z)$ of $M(S O(n))$ with integer coefficient is isomorphic to the ideal generated by the Euler-Poincaré class $X^{n}$ in the algebra $H^{*}\left(\hat{G}_{n}, Z\right)$ (Thom [18], Chapitre II, §5).

Henceiorth we identify the cohomology rings of $M(S O(n))$ and the abovementioned ideals of the cohomology rings of Grassmann manifold respectively.

6) The stable homotopy groups $\pi_{n+k}(M(S O(n)))$ are finite if $k \neq 0, \bmod 4$; the free components of the stable homotopy groups $\pi_{n+4 j}(M(S O(n)))$ are of rank $\pi(j)$, where $\pi(j)$ is the number of partition of $j$ (Thom [18], Théorème IV. 15).

To our purpose, therefore, it is sufficient to calculate the $p$-primary components of $\pi_{n+k}(M(S O(n)))$ for each prime $p$.

B. 2-primary components of $\pi_{n+k}(M(S O(n)))$

We will calculate the 2-primary components of $\pi n+k(M(S O(n)))$ for $8 \leqq k \leqq 12$. Let $Y_{2}$ be the product of the Eilenberg-MacLane complexes :

$$
\begin{aligned}
Y_{2} & =K(Z, n) \times K(Z, n+4) \times K\left(Z_{2}, n+5\right) \times(K(Z, n+8))^{2} \\
& \times\left(K\left(Z_{2}, n+9\right)\right)^{2} \times K\left(Z_{2}, n+10\right) \times K\left(Z_{2}, n+11\right) \times(K(Z, n+12))^{3} .
\end{aligned}
$$

Let

$$
\begin{array}{ll}
f_{1}: M(S O(n)) \rightarrow K(Z, n), & f_{2}: M(S O(n)) \rightarrow K(Z, n+4), \\
f_{3}: M(S O(n)) \rightarrow K\left(Z_{2}, n+5\right), & f_{4}: M(S O(n)) \rightarrow K(Z, n+8), \\
f_{5}: M(S O(n)) \rightarrow K(Z, n+8), & f_{6}: M(S O(n)) \rightarrow K\left(Z_{2}, n+9\right),
\end{array}
$$




$$
\begin{aligned}
& f_{i}: M(S O(n)) \rightarrow K\left(Z_{2}, n^{2}+9\right), \quad f_{8}: M(S O(n)) \rightarrow K\left(Z_{2}, n+10\right), \\
& f_{9}: M(S O(n)) \rightarrow K\left(Z_{2}, n+11\right), \quad f_{10}: M(S O(n)) \rightarrow K(Z, n+12), \\
& f_{11}: M(S O(n)) \rightarrow K(Z, n+12), \quad f_{12}: M(S O(n)) \rightarrow K(Z, n+12),
\end{aligned}
$$

be mappings defined by

$$
\begin{array}{ll}
f_{1}^{*}\left(\iota_{n}\right)=X^{n}, & f_{2}^{*}\left(\iota_{n+1}\right)=X^{n} P^{4}, \\
f_{3}^{*}\left(\iota_{n+5}\right)=W^{n} W^{3} W^{2}, & f_{4}^{*}\left(\iota_{n+8}\right)=X^{n} P^{8}, \\
f_{5}^{*}\left(\iota_{n+8}^{\prime}\right)=X^{n}\left(P^{4}\right)^{2}, & f_{6}^{*}\left(\iota_{n+9}\right)=W^{n} W^{5} W^{4}, \\
f_{7}^{*}\left(\iota_{n+9}^{\prime}\right)=W^{n} W^{3}\left(W^{2}\right)^{3}, & f_{8}^{*}\left(\iota_{n+10}\right)=W^{n} W^{6} W^{4}, \\
f_{9}^{*}\left(\iota_{n+11}\right)=W^{n} W^{5}\left(W^{2}\right)^{3}, & f_{10}^{*}\left(\iota_{n+12}\right)=X^{n} P^{12}, \\
f_{11}^{*}\left(\iota_{n+12}^{\prime}\right)=X^{n} P^{8} P^{4}, & f_{12}^{*}\left(\iota_{n+12}^{\prime \prime}\right)=X^{n}\left(P^{4}\right)^{3},
\end{array}
$$

respectively, where $\iota_{n+i}, \iota_{n+i}^{\prime}$ and $\iota_{n+12}^{\prime \prime}$ are the fundamental classes of the corresponding Eilenberg-MacLane complexes.

We define $F: M(S O(n)) \rightarrow Y_{2}$ by $f \circ d$ where $d$ is the diagonal map $M(S O(n)) \rightarrow(M(S O(n)))^{12}$ and $f=\prod_{i=1}^{12} f_{i}:(M(S O(n)))^{12} \rightarrow Y_{2}$.

Let us calculate the homomorphism $F^{*}$ induced by $F$

$$
F^{*}: H^{*}\left(Y_{2}, Z_{2}\right) \rightarrow H^{*}\left(M(S O(n)), Z_{2}\right) \text {. }
$$

We consider $F^{*}$ for the dimension $i \leqq n+13$. It has been verified by Thom [18] that $F^{*}$ is an isomorphism of $H^{i}\left(Y_{2}, Z_{2}\right)$ onto $H^{i}\left(M(S O(n)), Z_{2}\right)$ for $i \leqq n+7$. We can verify further that $F^{*}: H^{i}\left(Y_{2}, Z_{2}\right) \rightarrow H^{i}\left(M(S O(n)), Z_{2}\right)$ is an isomorphism into for $i \leqq n+13$, and a homomorphism onto for $i<n+13$ by continuing the analogous calculation as Thom ([18], Chapitre II, $\S 8 ; \S 1 . \mathbf{B}$, $\S 1 . \mathbf{D}, \S 2 . \mathbf{A})$. From this we deduce by Serre's $C$-theory $(\S 1 . \mathbf{C})$ that $F_{*}: \pi_{i}(M(S O(n))) \rightarrow \pi_{i}\left(Y_{2}\right)$ are $C_{2}$-isomorphisms for $i<n+13$ and $C_{2}$-onto for $i \leqq n+13$. Thus we have:

$$
\begin{aligned}
& \pi_{n+8}(M(S O(n))) \cong Z+Z, \bmod C_{2}, \\
& \pi_{n+9}(M(S O(n))) \cong Z_{2}+Z_{2}, \bmod C_{2} . \\
& \pi_{n+10}(M(S O(n))) \cong Z_{2}, \bmod C_{2}, \\
& \pi_{n+11}(M(S O(n))) \cong Z_{2}, \bmod C_{2}, \\
& \pi_{n+12}(M(S O(n))) \cong Z+Z+Z, \bmod C_{2} .
\end{aligned}
$$

Consequently we obtain

Proposition 1. The 2-primary components of the stable homotopy groups $\pi_{n+k}(M(S O(n)))$, for $8 \leqq k \leqq 12$, are: 


$$
\begin{array}{rcccr}
0, & Z_{2}+Z_{2}, & Z_{2}, & Z_{2}, & 0, \\
\text { for } k=8, & 9, & 10, & 11, & 12,
\end{array}
$$

respectively.

\section{C. p-primary components of $\pi n+k(M(S O(n)))$}

Let $p$ be an odd prime. The calculations of the $p$-primary components of the stable homotopy groups $\pi_{n+k}(M(S O(n)))$ are a little more complicated than 2-primary components.

There exists an aspherical fibre space $A$ with the fibre $K(Z, n+2 p-2)$ over the base $K(Z, n+2 p-1)$ (Serre [12]). Consider a mapping $\varphi$ of the complex $K(Z, n)$ into $K(Z, n+2 p-1)$ such that $\varphi^{*}\left(\ell_{n+2 p-1}\right)=\alpha_{p} \circ \operatorname{St}_{p}^{2 p-2}\left(\ell_{n}\right)$. We denote by $L(n, p)$ the fibre space induced from the fibre space $A$ by the mapping $\varphi$. So $L(n, p)$ is the fibre space over the complex $K(Z, n)$ of fibre $K(Z, n+2 p-2)$; non zero homotopy groups of $L(n, p)$ are only $\pi_{n}$ and $\pi_{n+2 p-2}$, both isomorphic to $Z$. The Eilenberg-MacLane invariant $\mathrm{k} \in H^{n+2 p-1}(Z, n ; Z)$ associated with $L(n, p)$ is $\alpha_{p} \circ S t_{p}^{2 p-2}\left(\ell_{n}\right)$.

Now we consider the cohomology mod $p$ of the complex $L(n, p)$ in dimension $<n+4 p-4$. It is necessaly to discuss the spectral sequence relative to the fibering of $L(n, p)$ over $K(Z, n)$. By the construction of $L(n, p), \iota_{n+2 p-2}$ is mapped by the transgression $\tau$ (explicitly by $d_{n+2 p-1}$ ) to the class $\operatorname{St}_{p}^{2 p-1}\left(\iota_{n}\right)$. It follows that in total degree $\leqq n+4 p-5, E_{\infty}^{r, s}$ are zero except for the following terms:

$$
\begin{aligned}
& E_{\infty}^{0,0}=E_{2}^{0,0}=H^{0}\left(Z, n ; Z_{p}\right), \\
& E_{\infty}^{n, 0}=E_{2}^{n, 0}=H^{n}\left(Z, n ; Z_{p}\right), \\
& E_{\infty}^{n+2 \not p-2,0}=E_{2}^{n+2 p-2,0}=H^{n+2 p-2}\left(Z, n ; Z_{p}\right) .
\end{aligned}
$$

Consequently the cohomology groups $H^{i}\left(L(n, p), Z_{p}\right)$, for $i \leqq n+4 p-5$, admit only the following generators:

$$
\begin{array}{ll}
\text { in dimension } n, & \bar{\iota}_{n}=p^{*}\left(\iota_{n}\right), \\
\text { in dimension } n+2 p-2, & S t_{p}^{2 p-2}\left(\bar{\iota}_{n}\right)=p^{*}\left(S t_{p}^{2 p-2}\left(\iota_{n}\right)\right),
\end{array}
$$

where $p: L(n, p) \rightarrow K(Z, n)$ is the projection (cf. Serre [12], p. 456).

Let $Y_{p}$ be the product of the complex $L(n, p)$ and the Eilenberg-MacLane complexes :

$$
\begin{aligned}
Y_{p}=L(n, p) \times K(Z, n+4) \times \ldots \times(K(\dot{Z}, n+4 h))^{\pi(h)} \times \\
\ldots \times(K(Z, n+2 p-6))^{\pi\left(\left(p-3^{\prime} / 2\right)\right.} \times(K(Z, n+2 p-2))^{\pi((p-1) / 2)-1} .
\end{aligned}
$$


Let us define a mapping $G: \dot{M}(S O(n)) \rightarrow Y_{p}$. There exists a mapping $g$ of $M(S O(n))$ into $K(Z, n)$ such that $g^{*}\left(e_{n}\right)=X^{n}$. And there exists a mapping $s$ of the $(n+2 p-2)$-skeleton of $K(Z, n)$ into $L(n, p)$ such that $s_{*}: \pi_{i}\left(K(Z, n)^{n+2 p-2}\right)$ $\rightarrow \pi_{i}(L(n, p))$ is an isomorphism onto for $i \leqq n+2 p-3$. So we have the mapping $s \circ g$ of the $(n+2 p-2)$-skeleton of $M(S O(n))$ into $L(n, p)$ such that $(s \circ g)\left(\bar{t}_{n}\right)=X^{n}$. The obstruction to the extension of the mapping $s$ is given by $\alpha_{p} \circ \operatorname{St}_{p}^{2 p-2}\left({ }_{\ell n}\right)$, and therefore, the obstruction to the extension of the mapping $s \circ g$ is

$$
c(s \circ g)=g^{*}(c(s))=\alpha_{p} \circ \operatorname{St}_{p}^{2 p-2}\left(X^{\prime \prime}\right) .
$$

Since $H^{*}(M(S O(n)), Z)$ has no $p$-torsion, we have $c(s \circ g)=0$. So we can extend the mapping $s \circ \mathrm{g}$ to a mapping $g: M(S O(n)) \rightarrow L(n, p)$ such that $\bar{g}^{*}\left(\bar{\iota}_{n}\right)=X^{n}$.

Now we introduce in the free base of $H^{n+1 i t}(M(S O(n)), Z)$ the lexicographic order using the dimension of Pontrjagin classes:

$$
\begin{aligned}
& X^{n} P^{1 h_{1}} P^{1 h_{2}} \ldots P^{! h_{r}}>X^{n} P^{1 k_{1}} P^{4 k_{2}} \ldots P^{1 k_{s}}, \\
& \text { if } \quad h_{1} \geqslant h_{2} \geqslant \ldots \geqslant h_{r}, k_{1} \geqslant k_{2} \geqslant \ldots \geqslant k_{s}, \\
& \quad h_{1}=k_{1}, \ldots, h_{t}=k_{t}, h_{t+1}>h_{t+1} .
\end{aligned}
$$

For example in $H^{n+12}(M(S O(n)), Z)$, we have: $X^{n} P^{12}>X^{n} P^{n} P^{1}>X^{n}\left(P^{1}\right)^{3}$. We denote by $K_{i}(Z, n+4 h)$ the $i$-th copy of $K(Z, n+4 h)$ in the corresponding factor of the product space $Y_{p}$, and by $i_{n+4 h}^{i}$ the fundamental class of $K_{i}(Z, n+4 h)$. There exist mappings

$$
\begin{aligned}
g_{h}^{i}: M(S O(n)) \rightarrow K_{i}(Z, n+4 h), & 0<h \leqq \frac{p-1}{2}, \quad 1 \leqq i \leqq \pi(h), \\
& (h, i) \neq\left(\begin{array}{c}
p-1 \\
2
\end{array}, \pi\left(\begin{array}{c}
p-1 \\
2
\end{array}\right)\right),
\end{aligned}
$$

such that $\left(g_{h}^{i}\right) *\left(c_{n+4 h}^{i}\right)=$ the $i$-th element of the free base of $H^{n+4 h}(M(S O(n)), Z)$.

Let $d: M(S O(n)) \rightarrow(M(S O(n)))^{m}, m=\sum_{n=1}^{(n-1) ! 2} \pi(h)$, be the diagonal map and $\bar{G}:(M(S O(n)))^{m} \rightarrow Y_{p}$ be

$$
\begin{aligned}
\bar{G}=g \times \Pi g_{h}^{i}, & \hat{0}<h \leqq \frac{p-1}{2}, \quad 1 \leqq i \leqq \pi(h), \\
& (h, i) \neq\left(\begin{array}{c}
p-1 \\
2
\end{array}, \pi\left(\begin{array}{c}
p-1 \\
2
\end{array}\right)\right) .
\end{aligned}
$$

We define $G: M(S O(n)) \rightarrow Y_{p}$, by the composed mapping $\bar{G} \circ d$.

We will calculate the homomorphism $G^{*}$ induced by $G$ 


$$
G^{*}: H^{n+j}\left(Y_{p}, Z_{p}\right) \rightarrow H^{n+j}\left(M(S O(n)), Z_{p}\right), \text { for } j \leqq 2 p+1 .
$$

In the first place we consider $G^{*}\left(\operatorname{St}_{p}^{2 b-2}\left(\bar{\ell}_{n}\right)\right)$ :

$$
\begin{aligned}
& G^{*}\left(\operatorname{St}_{p}^{2 p-2}\left(\bar{t}_{n}\right)\right)=\operatorname{St}_{p}^{2 p-2}\left(G^{*}\left(\bar{t}_{n}\right)\right)=\operatorname{St}_{p}^{2 p-2}\left(X^{n}\right) \\
& \quad=X^{n} \sum\left(x_{1}^{2}\right)^{(p-1) / 2}=X^{n}\left\{\left(\sum x_{1}^{2}\right)^{(p-1 / 2}+Q\left(x_{1}^{2}, x_{2}^{2}, \ldots, x_{n / 2}^{2}\right)\right\},
\end{aligned}
$$

where $Q\left(x_{1}^{2}, x_{2}^{2}, \ldots, x_{n / 2}^{2}\right)$ is a polynomial of elementary symmetric functions of $x_{i}^{2}$ which does not contain the term $\left(\sum x_{1}^{2}\right)^{(p-1) / 2}$ (cf. $\left.\S 1 . D\right)$. Now we have the next table:

$$
\begin{aligned}
& j=0, \\
& G^{*}\left(\bar{\iota}_{n}\right)=X^{n} \\
& j=4 h, 0<h<p-1, G^{*}\left(\iota_{n+4 h}^{i}\right)=\text { the } i \text {-th base element of } \\
& H^{n+4 h}\left(M(S O(n)), Z_{p}\right), \\
& \text { for } 1 \leqq i \leqq \pi(h), \\
& j=2 p-2, \quad \text { the } i \text {-th base element of } \\
& H^{n+2 p-2}\left(M(S O(n)), Z_{p}\right), \\
& \text { for } 1 \leqq i<\pi\left(\frac{p-1}{2}\right), \\
& G^{*}\left(S t_{p}^{2 p-2}\left(\bar{\imath}_{n}\right)\right)=X^{n}\left(P^{4}\right)^{(p-1) / 2}+\text { linear combi- } \\
& \text { nation of other base elements. }
\end{aligned}
$$

We know that the classes of $H^{k}\left(Y_{p}, Z_{p}\right)$ and $H^{k}\left(M(S O(n)), Z_{p}\right)$, which appear in the table, form bases of $H^{k}\left(Y_{p}, Z_{p}\right)$ and of $H^{k}\left(M(S O(n)), Z_{p}\right)$ respectively for $k \leqq n+2 p+1$; therefore we can easily verify that $G^{*}: H^{k}\left(Y_{p}, Z_{p}\right)$ $\rightarrow H^{k}\left(M(S O(n)), Z_{p}\right)$ is an isomorphism onto for $k \leqq n+2 p+1$. By Serre's $C$-theory we have that $G^{*}: \pi_{k}(M(S O(n))) \rightarrow \pi_{k}\left(Y_{p}\right)$ is $C_{p}$-isomorphism for $k<n+2 p+1$ and $C_{p}$-onto for $k \leqq n+2 p+1$. Thus we have:

$$
\begin{aligned}
& \pi_{n+k}(M(S O(n))) \cong 0, \bmod C_{p}, \text { if } k=0(4), k \leqq 2 p, \\
& \pi_{n+4 h}(M(S O(n))) \cong \underbrace{Z+Z+\ldots+Z}_{\pi(h)}, \bmod C_{p}, \text { if } 2 h<p .
\end{aligned}
$$

Consequently we obtain

Proposition 2. The p-primary components of the stable homotopy groups $\pi_{n+k}(M(S O(n)))$ are zero if $k \leqq 2 p$, where $p$ is an odd prime.

As a special case

Proposition 3. Let $p$ be a prime $\geq 7$. The p-primary components of tine stable homotopy groups $\pi_{n+k}(M(S O(n)))$ are zero for $k \leqq 14$, 


\section{5-primary components of $\pi_{n+k}(M(S O(n)))$}

Now we consider two Postnikov complexes $L(n, 5)$ and $L(n+4,5)$ defined above. As is mentioned in $\$ 2$. C, the cohomology groups $H^{i}\left(L(n, 5), Z_{5}\right)$ for $i \leqq n+15$ admit only the following generators:

in dimension $n, \quad \bar{\iota}_{n}=p_{1}^{*}\left(\iota_{n}\right)$,

in dimension $n+8, \quad S t_{5}^{8}\left(\bar{\iota}_{n}\right)=p_{1}^{*}\left(S t_{5}^{8}\left(\iota_{n}\right)\right)$,

where $p_{1}: L(n, 5) \rightarrow K(Z, n)$ is the projection of $L(n, 5)$. And we know also that the cohomology groups $H^{i}\left(L(n+4,5), Z_{5}\right)$ for $i \leqq n+15$ admit only the following generators:

in dimension $n+4, \quad \bar{\iota}_{n+4}=p_{2}^{*}\left(\iota_{n+4}\right)$,

in dimension $n+12, \quad S t_{5}^{8}\left(\bar{\iota}_{n+1}\right)=p_{2}^{*}\left(S t_{5}^{8}\left(\iota_{n+1}\right)\right)$,

where $p_{2}: L(n+4,5) \rightarrow K(Z, n+4)$ is the projection.

Let $Y_{5}$ be the product of $L(n, 5), L(n+4,5)$ and the Eilenberg-MacLane complexes :

$$
Y_{5}=L(n, 5) \times L(n+4,5) \times K(Z, n+8) \times(K(Z, n+12))^{2} .
$$

Let us define a mapping $H: M(S O(n)) \rightarrow Y_{5}$. By the same method as in $\S 2$. C, we can find a mapping $h_{1}$ of $M(S O(n))$ into $L(n, 5)$ such that $h_{1}^{*}\left(\bar{\iota}_{n}\right)$ $=X^{n}$, and a mapping $h_{2}$ of $M(S O(n))$ into $L(n+4,5)$ such that $h_{2}^{*}\left(\bar{\zeta}_{n+4}\right)$ $=X^{n} P^{4}$. On the other hand, there exist mappings

$$
\begin{array}{ll}
h_{3}: M(S O(n)) \rightarrow K(Z, n+8), & \text { such that } h_{3}^{*}\left(\ell_{n+8}\right)=X^{n} P^{8}, \\
h_{1}: M(S O(n)) \rightarrow K(Z, n+12), & \text { such that } h_{4}^{*}\left(\iota_{n+12}\right)=X^{n} P^{8} P^{1}, \\
h_{5}: M(S O(n)) \rightarrow K(Z, n+12), & \text { such that } h_{5}^{*}\left(\ell_{n+12}^{\prime}\right)=X^{n}\left(P^{4}\right)^{3} .
\end{array}
$$

We define $H: M(S O(n)) \rightarrow Y_{5}$ to be $h \circ d$, where $\left.d: M(S O(n)) \rightarrow M(S O(n))\right)^{5}$ is the diagonal map, and

$$
h=\prod_{i=1}^{5} h_{i}:(M(S O(n)))^{5} \rightarrow Y_{5} .
$$

We will calculate the homomorphism $H^{*}$ induced by $H$,

$$
\begin{aligned}
& H^{*}: H^{n+i}\left(Y_{5}, Z_{5}\right) \rightarrow H^{n+i}\left(M(S O(n)), Z_{5}\right), \text { for } i \leqq 15 . \\
& i=0, \quad H^{*}\left(\bar{\iota}_{n}\right)=X^{n}, \\
& i=4, \quad H^{*}\left(\bar{\zeta}_{n+4}\right)=X^{n} P^{4}
\end{aligned}
$$




$$
\begin{array}{ll}
i=8, & H^{*}\left(S t_{5}^{8} \tau_{n}\right)=3 X^{n} P^{4}+X^{n}\left(P^{4}\right)^{2}, \\
& H^{*}\left(\iota_{n+8}\right)=X^{n} P^{8}, \\
i=12, & H^{*}\left(S t_{5}^{8} \check{\iota}_{n+4}\right)=X^{n} P^{12}+2 X^{n} P^{8} P^{4}+3 X^{n}\left(P^{4}\right)^{3}, \\
& H^{*}\left(\iota_{n+12}\right)=X^{n} P^{8} P^{4} \\
& H^{*}\left(\iota_{n+12}^{\prime}\right)=X^{n}\left(P^{4}\right)^{3}
\end{array}
$$

For the present section $2 p-2=8$, so the classes of $H^{*}\left(Y_{5}, Z_{5}\right)$ and $H^{*}\left(M(\mathrm{~S} O(n)), Z_{5}\right)$ written in the table form bases of $H^{i}\left(Y_{5}, Z_{5}\right)$ and $H^{i}\left(M(S O(n)), Z_{5}\right)$. Therefore we can easily verify that $H^{*}: H^{i}\left(Y_{5}, Z_{5}\right)$ $\rightarrow H^{i}\left(M(S O(n)), Z_{5}\right)$ is an isomorphism onto for $i \leqq n+15$. By the $C$-theory we have that $H_{*}: \pi_{i}(M(S O(n))) \rightarrow \pi_{i}\left(Y_{5}\right)$ is $C_{5}$-isomorphism for $i<n+15$, and $C_{5}$-onto for $i \leqq n+15$; namely

$$
\begin{aligned}
& \pi_{n+8}(M(S O(n))) \cong Z+Z, \bmod C_{5}, \\
& \pi_{n+12}(M(S O(n))) \cong Z+Z+Z, \bmod C_{5}, \\
& \pi_{n+i}(M(S O(n))) \cong 0, \bmod C_{5}, \text { for } 8<i<12,12<i<15 .
\end{aligned}
$$

Thus we have

Proposition 4. The 5-primary components of the stable homotopy groups $\pi n+i(M(S O(n)))$, for $8 \leqq i \leqq 14$, are all zero.

E. 3-primary components of $\pi_{n+k}(M(S O(n)))$

The calculation of the 3-primary components of the stable homotopy groups $\pi_{n+k}(M(S O(n)))$ is the most complicated.

In the first place we consider the cohomology groups mod 3 of the Postnikov complex $L(n, 3)$ in the dimension $\leqq n+15$. We discuss the spectral sequence $\bmod 3$ relative to the fibering of $L(n, 3)$ over $K(Z, n)$. By the construction of $L(n, 3)$ the fundamental class $i_{n+1}$ of fibre is mapped by the transgression $\tau$ (explicitly $d_{n+5}$ ) onto the class $S t_{3}^{5}\left(\iota_{n}\right)$. As the reduced powers commute with the transgression, the calss $S t_{3}^{4}\left(\iota_{n+5}\right)$ of fibre is mapped by $\tau$ to the class $S t_{3}^{4} \circ S t_{3}^{5}\left(\iota_{n}\right)=S t_{3}^{9}\left(\iota_{n}\right)$ and the class $S t_{3}^{8}\left(\iota_{n+5}\right)$ to the class $S t_{3}^{8} \circ S t_{3}^{5}\left(\iota_{n}\right)$ $=S t_{3}^{13}\left(\iota_{n}\right)$ (cf. $\left.\S 1 . \mathrm{A}\right)$. It follows that in total degree $\leqq n+15, E_{\infty}^{r, s}$ are zero except for the following terms :

$$
\begin{array}{ll}
E_{\infty}^{0,0}=E_{2}^{0,0}=H^{0}\left(Z, n ; Z_{3}\right), & E_{\infty}^{n, 0}=E_{2}^{n, 0}=H^{n}\left(Z, n ; Z_{3}\right), \\
E_{\infty}^{n+4,0}=E_{2}^{n+4,0}=H^{n+4}\left(Z, n ; Z_{3}\right), & E_{\infty}^{n+8,0}=E_{2}^{n+8,0}=H^{n+8}\left(Z, n ; Z_{3}\right), \\
E_{\infty}^{0, n+9}=E_{2}^{0, n+9}=H^{n+9}\left(Z, n+4 ; Z_{3}\right), & E_{\infty}^{n+12,0}=E_{2}^{n+12,0}=H^{n+12}\left(Z, n ; Z_{3}\right), \\
E_{\infty}^{0, n+13}=E_{2}^{0, n+13}=H^{n+13}\left(Z, n+4 ; Z_{3}\right) . &
\end{array}
$$


Consequently the cohomology groups $H^{2}\left(L(n, 3), Z_{3}\right)$, for $i \leqq n+15$, admit only the following generetors:

$$
\begin{array}{ll}
\text { in dimension } n, & \bar{\iota}_{n}=p_{1}^{*}\left(\iota_{n}\right), \\
\text { in dimension } n+4, & S t_{3}^{4}\left(\bar{\iota}_{n}\right), \\
\text { in dimension } n+8, & S t_{3}^{8}\left(\bar{\iota}_{n}\right), \\
\text { in dimension } n+9, & \left(i_{1}^{*}\right)^{-1} \circ S t_{3}^{5}\left(\iota_{n+4}\right), \\
\text { in dimension } n+12, & S t_{3}^{12}\left(\bar{\iota}_{n}\right), \\
\text { in dimension } n+13, & \left(i_{1}^{*}\right)^{-1} \circ S t_{3}^{9}\left(\iota_{n+1}\right),
\end{array}
$$

where $p_{1}$ is the projection of $L(n, 3)$ into $K(Z, n)$ and $i_{1}: K(Z, n+4) \rightarrow L(n, 3)$ is the injection of fibre in the total space (cf. Serre [12], p. 456).

We need a certain class to construct the fibre space over $L(n, 3)$ which is indispensable to our purpose.

Lemma 1. There exists an integral cohomology class $u \in H^{n+9}(L(n, 3), Z)$ such that

i) $\rho_{3}(u)=\left(i_{1}^{*}\right)^{-1} \circ \mathrm{St}_{3}^{5}\left(\iota_{n+1}\right)$,

ii) $3^{m} u=0$, for a certain integer $m$,

where $\rho_{3}$ is the reduction $\bmod 3$.

Proof. The following diagram is commutative :

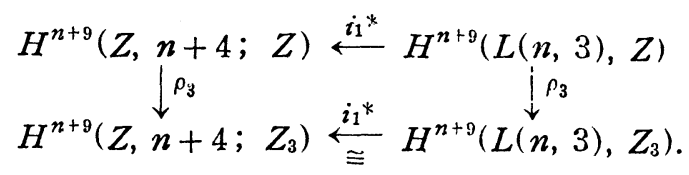

So it is sufficient to show that $\operatorname{St}_{3}^{5}\left(\iota_{n+4}\right) \in H^{n+9}\left(Z, n+4 ; Z_{3}\right)$ is the image by $\rho_{3} \circ i_{1}^{*}$ of a class $u \in H^{n+9}(L(n, 3), Z)$ satisfying the condition ii). Now we factorize the homomorphism $i_{1}^{*}$ with use of the spectral sequence associated with the fibering $L(n, 3)$ :

$$
\begin{aligned}
H^{n+9}(Z, n+4 ; Z) & =E_{2}^{0, n+9}=E_{n+10}^{0, n+9} \stackrel{\lambda}{\longleftarrow} E_{n+11}^{0, n+9}=E_{\infty}^{0, n+9} \\
=D^{0, n+9} / D^{1, n+8} & =D^{0, n+9} / E_{\infty}^{n+9,0} \longleftarrow D^{0, n+9}=H^{n+9}(L(n, 3), Z),
\end{aligned}
$$

where $\lambda$ is an isomorphism into and $\mu$ is a homomorphism onto (cf. Serre [12], p. 456). The term $E_{n+11}^{0, n+9}$ is the subgroup of $E_{n+10}^{0, n+9}$ consisting of $d_{n+10}$-cocycles :

$$
E_{n+10}^{0, n+9} \stackrel{d_{n+10}}{\longrightarrow} E_{n+10}^{n+10,0}=E_{2}^{n+10,0}=H^{n+10}(Z, n ; Z) .
$$


We know $H^{n+10}(Z, n ; Z)$ have no component but 2-primary one, and therefore for any element $x$ of $E_{n+10}^{0, n+9}, 2^{h} x$ is a $d_{n+10}$-cocycle and belongs to $E_{n+11}^{0, n+9}$ for a certain integer $h$. Consequently we can easily find an integer $i>0$ and an element $u^{\prime}$ such that

a) $2^{i} \cdot \alpha_{3} \circ S t_{3}^{4}\left(\iota_{n+4}\right)=\lambda\left(u^{\prime}\right), \quad u^{\prime} \in E_{n+11}^{0, n+9}$,

b) $2^{i} \equiv 1, \quad \bmod 3$.

We know that $H^{n+9}(Z, n+4 ; Z)$ has no components but 2- and 3-primary ones, and $H^{n+9}(L(n, 3), Z)$ has no components but 2-, 3-, and 5-primary ones. Since $3 \lambda\left(u^{\prime}\right)=0$, we have $3 u^{\prime}=0$, therefore we can find a class $u \in H^{n+9}(L(n, 3), Z)$ such that

i) $\mu(u)=u^{\prime}$,

ii) $3^{m} u=0$, for a certain integer $m$,

Thus the lemma is proved.

Let $A$ be an aspherical fibre space over the complex $K(Z, n+9)$ of fibre $K(Z, n+8)$. There exists a mapping $\varphi$ of the complex $L(n, 3)$ in $K(Z, n+9)$ such that $\varphi^{*}\left({ }_{(n+9}\right)=u$. We denote by $K_{3}$ the fibre space induced from the fibre space $A$ by the mapping $\varphi$. The non-zero homotopy groups of $K_{3}$ are only $\pi_{n}$, $\pi_{n+4}$ and $\pi_{n+8}$, all isomorphic to $Z$.

Now we consider the spectral sequence mod 3 relative to the fibering of $K_{3}$ over $L(n, 3)$. By the construction of $K_{3}$ the fundamental class $\imath_{n+8}$ of fibre is mapped by the transgression $\tau$ (explicitly $\left.d_{n+9}\right)$ onto the class $\left(i_{1}^{*}\right)^{-1} \circ S t_{3}^{5}\left(\ell_{n+4}\right)$, which is the generator of $H^{n+9}\left(L(n, 3), Z_{3}\right)$ (Lemma 1 ).

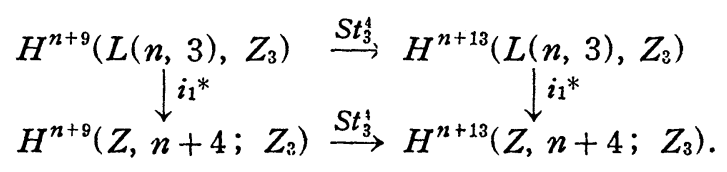

This diagram is commutative and the vertical homomorphisms are both isomorphisms onto. Consequently $\operatorname{St}_{3}^{4}\left(\ell_{n+8}\right)$ is mapped by the transgression to

$$
\begin{aligned}
S t_{3}^{4} \circ\left(i_{1}^{*}\right)^{-1} \circ S t_{3}^{5}\left(\iota_{n+1}\right) & =\left(i_{1}^{*}\right)^{-1} \circ S t_{3}^{4} \circ S t_{3}^{5}\left(\iota_{n+8}\right) \\
& =\left(i_{1}^{*}\right)^{-1} \circ S t_{3}^{9}\left(\iota_{n+8}\right) .
\end{aligned}
$$

It follows that in total degree $\leqq n+15, E_{\infty}^{r, s}$ are zero except for the following terms : 


$$
\begin{aligned}
& E_{\infty}^{0,0}=E_{2}^{0,0}=H^{0}\left(L(n, 3), Z_{3}\right), \quad E_{\infty}^{n, 0}=E_{2}^{n, 0}=H^{n}\left(L(n, 3), Z_{3}\right), \\
& E_{\infty}^{n+4,0}=E_{2}^{n+4,0}=H^{n+1}\left(L(n, 3), Z_{3}\right), \\
& E_{\infty}^{n+8,0}=E_{2}^{n+8,0}=H^{n+8}\left(L(n, 3), Z_{3}\right), \\
& E_{\infty}^{n+12,0}=E_{2}^{n+12,0}=H^{n+12}\left(L(n, 3), Z_{3}\right), \\
& E_{\infty}^{0, n+13}=E_{2}^{0, n+13}=H^{n+13}\left(Z, n+8 ; Z_{3}\right) .
\end{aligned}
$$

Consequently the cohomology groups $H^{i}\left(K_{3}, Z_{3}\right)$ for $i \leqq n+15$ admit only the following generators :

$$
\begin{array}{ll}
\text { in dimension } n, & \overline{\bar{t}}_{n}=p_{2}^{*}\left(\bar{\iota}_{n}\right), \\
\text { in dimension } n+4, & S t_{3}^{4}\left(\bar{\iota}_{n}\right), \\
\text { in dimension } n+8, & S t_{3}^{8}\left(\bar{\zeta}_{n}\right), \\
\text { in dimension } n+12, & S t_{3}^{12}\left(\bar{\zeta}_{n}\right), \\
\text { in dimension } n+13, & \left(i_{2}^{*}\right)^{-1} \circ S t_{3}^{5}\left(\ell_{n+8}\right),
\end{array}
$$

where $p_{2}$ is the projection of the fibre space $K_{3}$ and $i_{2}: K(Z, n+8) \rightarrow K_{3}$ is the injection of fibre in the total space $K_{3}$.

To eliminate the cohomology groups of dimension $n+13$, we will construct a fibre space over $K_{3}$ of fibre $K(Z, n+12)$.

Lemma 2. There exists an integral cohomology class $v \in H^{n+13}\left(K_{3}, Z\right)$ such that

i) $3^{m} v=0$, for an integer $m$,

ii) $\rho_{3}(v)=\left(i_{2}^{*}\right)^{-1} \circ S t_{3}^{5}\left(\iota_{n+8}\right)$.

Proof. We know that $H^{n+14}(L(n, 3), Z)$ has no components but 2-primary one, that $H^{n+13}(Z, n+8 ; Z)$ has no components but 2- and 3-primary ones, and that $H^{n+13}\left(K_{3}, Z\right)$ has no components but 2-, 3-, 5- and 7-primary ones. Therefore we can prove Lemma 2 by the same method as Lemma 1.

Let $B$ be an aspherical fibre space over $K(Z, n+13)$ of fibre $K(Z, n+12)$. There exists a mapping $\psi$ of the complex $K_{3}$ in $K(Z, n+13)$ such that $\psi^{* *}\left(\iota_{n+13}\right)$ $=v$. We denote by $K_{1}$ a fibre space induced from the fibre space $B$ by the mapping $\psi$. The non zero homotopy groups of $K_{4}$ are only $\pi_{n}, \pi_{n+4}, \pi_{n+8}$ and $\pi n+12$, all isomorphic to $Z$.

Now we consider the spectral sequence $\bmod 3$ relative to the fibering of $K_{1}$ over $K_{3}$. By the construction of $K_{4}$ the fundamental class ${ } n+12$ of fibre is mapped by the transgression : (explicitly $\left.d_{n+13}\right)$ onto the class $\left(i_{2}^{*}\right)^{-1} \circ S t_{3}^{5}\left(\ell_{n+s}\right)$ 
(Lemma 2). It follows that in total degree $\leqq n+15, E_{\infty}^{r, s}$ are zero except for the following terms :

$$
\begin{array}{ll}
E_{\infty}^{0,0}=H^{0}\left(K_{3}, Z_{3}\right), & E_{\infty}^{n, 0}=H^{n}\left(K_{3}, Z_{3}\right), \\
E_{\infty}^{n+4,0}=H^{n+4}\left(K_{3}, Z_{3}\right), & E_{\infty}^{n+8,0}=H^{n+8}\left(K_{3}, Z_{3}\right), \\
E_{\infty}^{n+12,0}=H^{n+12}\left(K_{3}, Z_{3}\right) . &
\end{array}
$$

Consequently the cohomology groups $H^{i}\left(K_{4}, Z_{3}\right)$ for $i \leqq n+15$ admit only the following generators:

$$
\begin{array}{ll}
\text { in dimension } n, & \widetilde{\imath}_{n}=p_{3}^{*}\left(\overline{\mathfrak{l}}_{n}\right), \\
\text { in dimension } n+4, & S t_{3}^{4}\left(\widetilde{\imath}_{n}\right), \\
\text { in dimension } n+8, & S t_{3}^{8}\left(\widetilde{\imath}_{n}\right), \\
\text { in dimension } n+12, & S t_{3}^{12}\left(\widetilde{\imath}_{n}\right),
\end{array}
$$

where $p_{3}$ is the projection of $K_{4}$.

On the other hand, as is shown in $\$ 2 . \mathbf{C}$, the cohomology groups $H^{i}\left(L(n+8,3), Z_{3}\right)$ of the Postnikov complex $L(n+8,3)$ for $i \leqq n+15$ admit only the following generators:

$$
\begin{array}{ll}
\text { in dimension } n+8, & \bar{\iota}_{n+8}=p_{4}^{*}\left(\iota_{n+8}\right), \\
\text { in dimension } n+12, & S t_{3}^{4}\left(\bar{\iota}_{n+8}\right),
\end{array}
$$

where $p_{4}$ is the projection of the fibre space $L(n+8,3)$ over $K(Z, n+8)$.

Let $Y_{3}$ be the product of $K_{4}, L(n+8,3)$ and $K(Z, n+12)$. Let us define a mapping $F: M(S O(n)) \rightarrow Y_{3}$. As is shown in $\$ 2 . \mathbf{C}$, we can find a mapping $f_{1}$ of $M(S O(n))$ into $L(n, 3)$ such that $f_{1}^{*}\left(\bar{t}_{n}\right)=X^{n}$. We know that there exists a mapping $t$ of the $(n+8)$-skeleton of $L(n, 3)$ into $K_{3}$ such that $t_{*}: \pi_{i}\left(L(n, 3)^{n+8}\right)$ $\rightarrow \pi_{i}\left(K_{3}\right)$ is an isomorphism onto for $i<n+8$. So we obtain the mapping $t \circ f_{1}$ of the $(n+8)$-skeleton of $M(S O(n))$ into $K_{3}$ such that $\left(t \circ f_{1}\right)^{*}(\bar{t})=X^{n}$. The obstruction to the extension of the mapping $t \circ f_{1}$ is given by $f_{1}^{*}(u)$. Since $H^{*}(M(S O(n)), Z)$ has no 3-torsion, we have $f_{1}^{*}(u)=0$ (see Lemma 1). Now we have the mapping $f_{2}$ of $M(S O(n))$ into $K_{3}$ such that $f_{2}^{*}\left(\bar{\tau}_{n}\right)=X^{n}$. In virture of Lemma 2, we can find a mapping $f_{3}$ of $M(S O(n))$ into $K_{4}$ such that $f_{3}^{*}\left(\tilde{\iota}_{n}\right)$ $=X^{n}$ by the same method as above. Similarly we can find a mapping $f_{i}$ of $M(S O(n))$ into $L(n+8,3)$ such that $f_{4}^{*}\left(\bar{\tau}_{n+8}\right)=X^{n}\left(P^{4}\right)^{2}$. On the other hand there exists a mapping $f_{5}$ of $M(S O(n))$ into $K(Z, n+12)$ such that $f_{5}^{*}\left(\ell_{n+12}\right)$ $=X^{n}\left(P^{4}\right)^{3}$. Now we define a mapping $F: M(S O(n)) \rightarrow Y_{3}$ to be the composition 
of the diagonal map $d$ and $f_{3} \times f_{4} \times f_{5}$ :

$$
M(S O(n)) \underset{d}{\longrightarrow}(M(S O(n)))^{3} \underset{f_{3} \times f_{4} \times f_{5}}{\longrightarrow} Y_{3} .
$$

We will calculate the homomorphism $F^{*}$ induced by $F$

$$
\begin{array}{ll}
F^{*}: H^{i}\left(Y_{3}, Z_{3}\right) \rightarrow H^{i}\left(M(S O(n)), Z_{3}\right), \text { for } i \leqq n+15 . \\
i=n, & F^{*}\left(\tau_{n}\right)=X^{n}, \\
i=n+4, & F^{*}\left(S t_{3}^{4}\left(\tau_{n}\right)\right)=X^{n} P^{4}, \\
i=n+8, & F^{*}\left(S t_{3}^{8}\left(\tau_{n}\right)\right)=X^{n} P^{8}, \quad F^{*}\left(\tau_{n+8}\right)=X^{n}\left(P^{4}\right)^{2}, \\
i=n+12, & F^{*}\left(S t_{3}^{12}\left(\tau_{n}\right)\right)=X^{n} P^{12}, \\
& F^{*}\left(S t_{3}^{4}\left(\tau_{n+8}\right)\right)=X^{n} P^{8} P^{4}+2 X^{n}\left(P^{4}\right)^{3}, \\
& F^{*}\left(\iota_{n+12}\right)=X^{n}\left(P^{4}\right)^{3} .
\end{array}
$$

For the present case $2 p-2=4$, so the classes of $H^{i}\left(Y_{3}, Z_{3}\right)$ and $\left.H^{i}(M i S O(n)), Z_{3}\right)$ written above form bases of $H^{i}\left(Y_{3}, Z_{3}\right)$ and $H^{i}\left(M(S O(n)), Z_{3}\right)$ for $i \leqq n+15$. Therefore we can verify that $F^{*}: H^{i}\left(Y_{3}, Z_{3}\right) \rightarrow H^{i}\left(M(S O(n)), Z_{3}\right)$ is an isomorphism onto for $i \leqq n+15$. By Serre's $C$-theory, we obtain that $F_{*}: \pi_{i}(M(S O(n))) \rightarrow \pi_{i}\left(Y_{3}\right)$ is $C_{3}$-isomorphism for $i<n+15$ and $C_{3}$-onto for $i \leqq n+15$. This implies

$$
\begin{aligned}
& \pi_{n+8}(M(S O(n))) \cong Z+Z, \quad \bmod C_{3}, \\
& \pi_{n+12}(M(S O(n))) \cong Z+Z+Z, \quad \bmod C_{3}, \\
& \pi_{n+i}(M(S O(n))) \cong 0, \quad \bmod C_{3} \text { for } 8<i<12,12<i<15 .
\end{aligned}
$$

Thus we have

Proposition 5. The 3-primary components of the stable homotopy groups $\pi_{n+i}(M(S O(n)))$, for $8 \leqq i \leqq 14$, are all zero.

\section{F. Results}

Here we state the results obtained in this section $\S 2$.

ThEOREM 1. i) The stable homotopy groups $\pi_{n+i}(M(S O(n)))$ are for $8 \leqq i \leqq 14$ :

$$
\begin{array}{lll}
\pi_{n+8}=Z+Z, & \pi_{n+9}=Z_{2}+Z_{2}, & \pi_{n+10}=Z_{2}, \\
\pi_{n+11}=Z_{2}, & \pi_{n+12}=Z+Z+Z, & \pi_{n+13}=2 \text {-group }, \\
\pi_{n+14}=2 \text {-group. } & &
\end{array}
$$

ii) Let $p$ be an odd prime. For $i \leqq 2 p, \pi_{n+i}(M(S O(n)))$ has no p-primary components. 


\section{$\S 3$. The groups of cobordism $\Omega^{k}$}

In this section we determine the groups of cobordism $\Omega^{k}$ for $8 \leqq k \leqq 12$ and discuss some problems related to the cobordism classes.

All manifolds considered are to be compact, orientable and differentiable, unless otherwise stated.

\section{A. Thom algebra}

Here we state briefly the definition of Thom algebra $\Omega$ and the central results of Thom [18] concerning $\Omega$.

We define the sum $V^{n}+W^{n}$ of two disjoint oriented manifolds $V^{n}$ and $W^{n}$ of the same dimension as the union of $V^{n}$ and $W^{n}$. The sum is oriented in natural way. For an oriented manifold $V^{n}$ an oriented manifold $-V^{n}$ is defined as follows: $-V^{n}$ is identical with $V^{n}$ as manifold and has the orientation opposite to that of $V^{n}$. The product $V^{n} \times W^{m}$ of two oriented manifolds $V^{n}$, $W^{m}$ of any dimensions is the oriented cartesian product,

An oriented manifold $V^{n}$ is bounded, when there exists an oriented manifold with boundary, $X^{n+1}$, whose oriented boundary (with the orientation and differentiable structure induced from $X^{n+1}$ ) is identical with the given oriented manifold $V^{n}$. Two oriented manifolds $V^{n}$ and $W^{n}$ are called "cobordantes" when $V^{n}+\left(-W^{n}\right)$ is bounded. This is an equivalence relation and compatible with the operation,+- and $\varkappa$ defined above. The equivalence classes of $n$ dimensional oriented manifolds form an additive group $\Omega^{n}$ under the operation + and -, and its null element is the class of bounded manifolds. We call

$\Omega^{n}$ the group of cobordism of dimension $n$. The direct sum $\Omega=\sum_{n=0}^{\infty} \Omega^{n}$ becomes an anticommutative graded algebra under the operation,+- and $\times$ defined above.

The groups of cobordism $\Omega^{k}$ are related to the stable homotopy groups of Thom complex $M(S O(n))$ by Thom [18].

THEOREM. The groups of cobordism $\Omega^{k}$ of dimension $k$ are isomorphic to the stable homotopy groups of Thom complex $M(S O(n))$ :

$$
\Omega^{k}=\pi_{n+k}(M(S O(n))) \text {, for } k<n .
$$

The following results of Thom [18] are founded on this theorem.

1) The groups $\Omega^{k}$ are finite for $k \neq 0, \bmod 4$. The group $\Omega^{4 i}$ is the direct sum of $\pi(i)$ free cyclic groups and a finite group. 
2) For $k<8$, the groups $\Omega^{k}$ are :

$$
\begin{array}{ll}
\Omega^{0}=Z, & \Omega^{1}=\Omega^{2}=\Omega^{3}=0, \\
\Omega^{4}=Z, & \Omega^{5}=Z_{2}, \quad \Omega^{6}=\Omega^{i}=0 .
\end{array}
$$

\section{B. The groups of cobordism $\Omega^{k}$}

Thom's theorem and Theorem 1 give

THEOREM 2. i) For $8 \leqq k \leqq 14$, the groups $\Omega^{k}$ are:

$$
\begin{array}{llll}
\Omega^{5}=Z+Z, & \Omega^{9}=Z_{2}+Z_{2}, & \Omega^{10}=Z_{2}, & \Omega^{11}=Z_{2}, \\
\Omega^{12}=Z+Z+Z, & \Omega^{13}=2 \text {-group }, & \Omega^{14}=2 \text {-group. } &
\end{array}
$$

ii) Let $p$ be an odd prime. The p-primary components of $\Omega^{k}$ are zero for $k \leqq 2 p$.

Generators of $\Omega^{k}$.

We denote by $P C(i)$ the complex $i$-dimensional projective space, and by $P(m, n)$ the $(m+2 n)$-manifold defined by Dold [6]. We know that $P(m, n)$ is orientable if and only if $m \neq n, \bmod 2$ or $m=0$ (cf. Dold [6], C). We denote by $\left[V^{n}\right]$ the element of $\Omega$ which contains an $n$-manifold $V^{n}$.

i) Generators of $\Omega^{S}$ are given by $[P C(4)]$ and $[P C(2) \times P C(2)]$.

ii) Generators of $\Omega^{9}$ are given by $[P(1,4)]$ and $[P(1,2) \times P C(2)]$, where $[P(1,2)]$ is the generator of $\Omega^{5}=\mathfrak{M}^{5}=Z_{2}$ given by $\mathrm{Wu}[19]$.

iii) The generator of $\Omega^{10}$ is $[P(1,2) \times P(1,2)]$.

iv) The generator of $\Omega^{11}$ is $[P(3,4)]$.

v) Generators of $\Omega^{12}$ are given by $[P C(6)],[P C(4) \times P C(2)]$ and $\left[(P C(2))^{3}\right]$.

As is shown above, $\Omega^{S}$ and $\Omega^{12}$ are free groups, therefore i) and v) are trivial (Thom [18], Chapitre IV, §8); ii)-iv) are the direct consequences of Theorem 2 and Dold [6] (Satz 3 and $H$ ).

Remark. Among the groups of cobordism $\Omega^{k}$ there exist no free groups but $\Omega^{4}, \Omega^{\Im}$ and $\Omega^{12}$, because for $i \geqq 4, \Omega^{4 i}$ has 2 -torsion $\left[V^{4 i}\right]$, where

$$
V^{4 i}=P C(2 i-8) \times P(3,4) \times P(1,2) .
$$

Now we consider the problem of Steenrod: What algebraic conditions are necessary and sufficient for an orientable manifold $V^{n}$ to be bounded? (Eilenberg [7], Problem 26). Rohlin and Thom have given partial answers for this problem ([10], [11], [18], Chapitre IV, §8); 
i) $V^{n}$ is always bounded for $n=1,2,3,6,7$;

ii) $V^{4}$ is bounded if and only if its index $\tau\left(V^{4}\right)$ is zero.

iii) $V^{5}$ is bounded if and only if its Stiefel-Whitney number $W^{3} W^{2}$ is zero.

We apply Theorem 2 to this problem.

Theorem 3. i) An 8-manifold is bounded if and only if all its Pontrjagin numbers are zero.

ii) A 9-manifold is bounded if and only if both its Stiefel-Whitney numbers $W^{3}\left(W^{2}\right)^{3}$ and $W^{7} W^{2}$ are zero.

iii) A 10-manifold is bounded if and only if its Stiefel-Whitney number $W^{6} W^{4}$ is zero.

iv) An 11-manifold is bounded if and only if its Stiefel-Whitney number $\left(W^{3}\right)^{3} W^{2}$ is zero.

v) A 12-manifold is bounded if and only if all its Pontrjagin numbers are zero.

Lemma 1. The Stiefel-Whitney class $^{3)}$ of the manifold $P(m, n)$ is given by

$$
W(m, n)=(1+c)^{m}(1+c+d)^{n+1}, \text { with } \quad c^{m+1}=0, \quad d^{n+1}=0,
$$

where $c$ and $d$ are 1- and 2-dimensional cohomology classes mod 2 respectively, and they generate $H^{*}\left(P(m, n), Z_{2}\right)$ (Dold [6], Satz 2).

Lemma 2. The Chern class $^{3)}$ of the complex projective space $P C(n)$ is equal to $(1+g)^{n+1}$, where $g$ is the generator of $H^{2}(P C(n), Z)$. The Pontrjagin class ${ }^{3)}$ of $P C(n)$ is equal to $\left(1+g^{2}\right)^{n+1}$ (Hirzebruch [8], Satz 4.10.2).

Proof of Theorem 3. For any 8-manifold $V^{8}$, we can describe $\left[V^{8}\right]$ $=a[P C(4)]+b[P C(2) \times P C(2)]$, where $a$ and $b$ are certain integers. As the Pontrjagin numbers are additive, $a$ and $b$ are uniquely determined by Lemma 2 :

$$
a=\frac{1}{5}\left(P^{4} P^{4}-2 P^{s}\right), \quad b=\frac{1}{9}\left(5 P^{8}-P^{4} P^{4}\right),
$$

where $P^{4} P^{4}$ and $P^{8}$ denote the corresponding Pontrjagin numbers of $V^{8}$. Therefore $\mathrm{i}$ ) is proved. Using Lemma 1 and Lemma 2 we can prove ii) $-\mathrm{v}$ ) by the same method as above.

Remark. The selections of Stiefel-Whitney numbers described in ii) and iv)

3) Precisely, we must say the Stiefel-Whitney polynomial with the variable $t=1$ (cf. Wu [21], p. 41) or total Stiefel-Whitney class. 
are not unique. It is evident from the proof that in iv), for example, we can take $W^{5}\left(W^{2}\right)^{3}$ instead of $\left(W^{3}\right)^{3} W^{2}$. However, in iii) no other Stiefel-Whitney numbers than $W^{6} W^{4}$ give a sufficient condition, because $W^{6} W^{4}$ is the unique Stiefel-Whitney number that is not zero for $P(1,2) \times P(1,2)$.

Especially we observe

CoROllary 1. For $n \leqq 12$, an $n$-sphere $S^{n}$ is bounded, even if it admits any differentiable structures."

Proof. For $n \neq 8,12$, it is trivial. For $n=8,12$, we know the following index formulas (Hirzebruch [8]) :

$$
\begin{aligned}
& 45 \tau=7 P^{8}-P^{4} P^{4}, \\
& 945 \tau=62 P^{12}-13 P^{8} P^{1}+2\left(P^{4}\right)^{3},
\end{aligned}
$$

where $\Pi P^{4 i}$ are the corresponding Pontrjagin numbers. Thus the corollary is the direct consequence of Theorem 3.

COROllary 2. For $n \leqq 11, n \neq 8$, the cobordism classes of $n$-manifolds $V^{n}$ are topological invariants, i.e., they are independent of their differentiable structures. ${ }^{4)}$

Proof. We know that the Stiefel-Whitney classes $W^{i}\left(V^{n}\right)$ of an $n$ dimensional manifold are topological invariants of $V^{n}$ (Thom [17]). Therefore the corollary is the immediate consequence of Theorem 3 .

Remark. If the Pontrjagin classes $P^{4 k}\left(V^{n}\right)$ of an $n$-dimensional manifold are topological invariants, Corollary 2 holds for $n=8$ and 12 .

\section{REFERENCES}

[1] M. Adachi. Sur les groupes de cobordisme $\Omega^{k}$, Proc. Japan Acad., 33 (1957), 143-144.

[2] A. Borel, La cohomologie modulo 2 de certaines espaces homogènes, Comment. Math. Helv., 27 (1953), 165-197.

[3] A. Borel-J. P. Serre, Groupes de Lie et puissances réduites de Steerod, Amer. J. Math., 75 (1953), 409-448.

[ 4 ] H. Cartan, Sur les groupes d'Eilenberg-MacLane $H(1 I, n)$, II, Proc. Nat. Acad. Sci. U.S.A., 40 (1954), 704-707.

[ 5 ] H. Cartan, Sur l'itération des opérations de Steenrod, Comment. Math. Helv., 29 (1955), 40-58.

4) No manifolds are known which can carry two distinct differentiable structures but 7-sphere, 15-sphere and certain 7- and 15-manifolds (cf. Milnor [9], Shimada [15] and Tamura [16]). 
[6] A. Dold, Erzeugende der Thomschen Algebra $\Re$, Math. Z., 65 (1956), 25-35.

[7] S. Eilenberg, On the problems of topology, Ann. of Math., 50 (1949), 247-260.

[8] F. Hirzebruch, Neue topologische Methoden in der algebraischen Geometrie, Springer, (1956).

[9] J. Milnor, On manifolds homeomorphic to the 7-aphere, Ann. of Math., 64 (1956), 399-405.

[10] B. Rohlin, Une variété de dimension 3 est le bord d'une variété de dimension 4, Doklady Akad. Nauk S.S.S.R., 81 (1951), 355-357.

[11] B. Rohlin, Nouveaux résultats en théorie des variétés de dimension 4, Doklady Akad. Nauk S.S.S.R., 84 (1952), 221-224.

[12] J. P. Serre, Homologie singulière des espaces fibrés. Applications, Ann. of Math., 54 (1951), 425-505.

[13] J. P. Serre, Groupes d'homotopie et classes de groupes abéliens, Ann. of Math., 58 (1953), 258-294.

[14] J. P. Serre, Cohomologie modulo 2 des complexes d'Eilenberg-MacLane, Comment. Math. Helv., 27 (1953), 198-231.

[15] N. Shimada, Differentiable structures on the 15-sphere and Pontrjagin classes of certain manifolds, Nagoya Math. J., 12 (1957), 59-69.

[16] I. Tamura, Homeomorphy classification of total spaces of sphere bundles over spheres, J. Math. Soc. Japan, 10 (1958), 29-43.

[17] R. Thom, Espaces fibrés en sphères et carrés de Steenrod, Ann. Sci. École Norm. Sup., 69 (1952), 109-182.

[18] R. Thom, Quelques propriétés globales des variétés différentiables, Comment. Math. Helv., 28 (1954), 17-86.

[19] W.-T. Wu, Classes caractéristiques et $i$-carrés d'une variété, C. R. Acad. Sci. Paris, 230 (1950), 508-511.

[20] W.-T. Wu, Les $i$-carrés dans une variété grassmannienne, C. R. Acad. Sci. Paris, 230 (1950), 918-920.

[21] W.-T. Wu, Sur les classes caractéristiques des structures fibrées sphériques, Actual. Sci. Ind., 1183 (1952), 1-89.

\section{Mathematical Institute}

Nagoya University 